\title{
On the mean-value theorem in an algebraic number field
}

\author{
By Yoshikazu EDA
}

\section{[1]}

(Received July 14, 1966)

Vinogradov's mean value theorem is very deep and fundamental in some problems

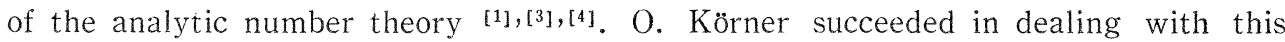
theorem in an algebraic number field, and he extended the generalized Waring problem for polynom sums to the case of number fields ${ }^{[6]}$. Using the Karacuba-Korobov ${ }^{[5]}$, we shall prove, in the present paper, the following main theorem.

Let $K$ be an algebraic number field of degree $n$. Let $K^{(l)}\left(1 \leqq l \leqq n_{1}\right)$ be $n_{1}$ real conjugate fields and $K^{(m)}, K^{\left(m+n_{2}\right)}\left(n_{1}+1 \leqq m \leqq n_{1}+n_{2}\right)$ be $n_{2}$ pairs of complex conjugate fields, so that $n_{1}+2 n_{2}=n$. We denote by $\gamma^{(i)}(1 \leqq i \leqq n)$ the conjugates of $\gamma \in K$. If $\xi=$ $\sum_{\nu=1}^{n} \gamma_{\nu} x_{\nu}$, then we define

$$
\xi^{(i)}=\sum_{\nu=1}^{n} \gamma_{\nu}^{(i)} x_{\nu}
$$

and

$$
\|\gamma\|=\operatorname{Max}_{1 \leqq i \leq n}\left|\gamma^{(i)}\right|,\|\xi-\gamma\|=\operatorname{Max}_{1 \leqq i \leqq n}\left|\xi^{(i)}-\gamma^{(i)}\right| .
$$

A number $\gamma$ of $K$ is called totally positive or totally non-negative according to

$$
\gamma^{(l)}>0 \text { or } \gamma^{(b)} \geq 0
$$

respectively. If $\gamma$ is a totally non-negative integer of $K$ and satisfies $\|\gamma\| \leqq T$ for some positive $T$, then we write

$$
0<\gamma<T \text {. }
$$

Let $\mathfrak{a}$ be the integral domain consisting of all algebraic integers in $K$. We use a letter $c$ (and similarly $c_{0}, c_{1}, \cdots \cdots$ ) to denote a positive constant depending only on $K$, not necessarily the same each time it occurs. The symbol

$$
f(x)=o(g(x)) \text { for } g(x) \neq 0
$$

means that $\lim _{x \rightarrow \infty} f(x) / g(x)=0$. [x] is the greatest integer in $x$. As usual, $a \mid b$ means that $a$ divides $b$, and $a \nmid b$ means that $a$ does not divide $b . a^{i} \| b$ means that $a^{i} \mid b$ but $a^{i+1}+b$. Let $k \geqq 2$.

Let $N_{s}\left(P ; \nu_{1}, \nu_{2}, \cdots \cdots, \nu_{k}\right)=N_{s}^{*}\left(Q ; \nu_{1}, \nu_{2}, \cdots \cdots, \nu_{k}\right)$, where $Q^{n}=P$, be the number of solutions of the system of $k$ Diophantine equations

with the conditions

$$
\left\{\begin{array}{c}
\lambda_{1}+\lambda_{2}+\cdots \cdots+\lambda_{s}=\mu_{1}+\mu_{2}+\cdots \cdots+\mu_{s}+\nu_{1}, \\
\lambda_{1}{ }^{2}+\lambda_{2}{ }^{2}+\cdots \cdots+\lambda_{s}{ }^{2}=\mu_{1}{ }^{2}+\mu_{2}{ }^{2}+\cdots \cdots+\mu_{s}{ }^{2}+\nu_{2}, \\
\cdots \cdots \cdots+\cdots \cdots+\lambda_{s}{ }^{k}=\mu_{1}{ }^{k}+\mu_{2}{ }^{k}+\cdots \cdots+\mu_{s}{ }^{k}+\nu_{k}, \\
\lambda_{1}{ }^{k}+\lambda_{2}{ }^{k}+\cdots \cdots \cdots+
\end{array}\right.
$$

$$
0<\lambda_{j}, \mu_{j}<Q, \quad \lambda_{j}, \mu_{j} \in \mathrm{0}
$$

$(1 \leqq j \leqq s)$,

where $k \geqq 2$ and $\nu_{1}, \nu_{2}, \cdots \cdots, \nu_{k}$ are arbitrary fixed integers in 0 . Let $N_{s}(P ; 0,0, \cdots \cdots, 0)$ $=N_{s}(P)=N_{s}^{*}(Q), Q^{n}=P$. 
Main Theorem. Let $0<\theta \leqq 1$ and $k \geqq 2$. $k$ denotes a positive integer. $R$ is a "sufficiently large" positive number with the condition that

(1) $R>\operatorname{Max}\left(k,\left(2^{n} \theta^{-1}\right)^{1 / k}\right)$.

$Q=Q_{0}$ is a positive real number with the condition that

$$
(2 R)^{k\left(1+\frac{1}{k-1}\right)^{\tau-1}}<Q=Q_{0}
$$

with a positive integer $\tau \geqq 1$ Let $Q_{0}{ }^{n}=P_{0}=P$. Further, let $s$ be a positive integer with

$$
s \geqq \frac{1}{2} k(k+1)+\tau k-1 .
$$

Then we have the following estimate:

where

$$
\begin{aligned}
N_{s}(P) & \leqq c^{\left(k^{2}+2 s+\frac{(\tau-1) s}{k}-\frac{1}{4}(\tau-1)(k+1)\right) \tau n}(1+2 \theta)^{2 s \tau+\frac{1}{k} \tau(\tau-1) s-\frac{1}{4} \tau(\tau-1)(k+1)} \\
& \times s^{2 k \tau} \cdot P^{2 s-\frac{1}{2} k(k-1)+\delta}
\end{aligned}
$$

$$
\delta=\delta(k)=\frac{1}{2} k(k+1)(1-1 / k)^{\tau} .
$$

This result can be described as the following form:

$$
\begin{aligned}
N_{s}(P) \leqq & c^{\left(k^{2}+2 s+\frac{(\tau-1)}{k} s-\frac{1}{2}(\tau-1)(k+1)\right) \tau(n+1)} s^{2 k \tau} \\
& \times P^{2 s-\frac{1}{2} k(k+1)+\frac{1}{2} k(k+1)(1-1 / k)^{\tau}} .
\end{aligned}
$$

I should like to express my indebtedness and gratitude to Professor Tikao Tatuzawa, now at University of Tokyo.

\section{[2] Lemmata.}

Lemma 1. If we denote by $\pi_{K}(x)$ the number of prime ideals $p$ of $K$, satisfying the condition $N p \leqq x$, then we have

$$
\pi_{K}(x)=\sum_{N \leqq x} 1=\frac{x}{\log x}+o\left(\frac{x}{\log x}\right),
$$

where $R \leqq x$.

Proof. This is the prime ideal theorem ${ }^{[7]}$.

Lemma 2. Let $R$ be a sufficiently large positive number. If for any real positive number $\varepsilon$, we have

$$
x, \frac{1}{1+\varepsilon} x \geqq R,
$$

then there exists at least one prime ideal $\mathfrak{p}$ in $K$ such that

$$
x>N \mathfrak{p} \geqq \frac{1}{1+\varepsilon} x .
$$

Proof. By Lemma 1 we have

$$
\begin{aligned}
\pi_{K}(x) & -\pi_{K}\left(\frac{1}{1+\varepsilon} x\right)=\sum_{\left.\frac{1}{1+\varepsilon} \leq N\right\}<x} 1 \\
= & \frac{x}{\log x}-\left(\frac{1}{1+\varepsilon} x\right) / \log \left(\frac{1}{1+\varepsilon} x\right)+o\left(\frac{x}{\log x}\right) \\
= & \frac{\varepsilon}{1+\varepsilon} \frac{x}{\log x}(1+o(1))>0,
\end{aligned}
$$

our lemma is accordingly proved. 
Lemma 3. Let a be a integral ideal of $K$. Then we can take a basis $\left[\alpha_{1}, \alpha_{2}, \cdots \cdots\right.$, $\alpha_{n}$ ] of $\mathfrak{a}$ such that

$$
c_{1} \sqrt[n]{N a} \leqq\left|\alpha_{(j)}{ }^{i}\right| \leqq c_{2} \sqrt[n]{N a} \quad(1 \leqq i, j \leqq n) .
$$

Proof [8]. Let $(5$ be the ideal class containing $\mathfrak{a}$. Then $\mathfrak{a}$ is a product of a fixed representative ideal $\mathfrak{b}$ in $\mathfrak{s}$ and a number $\rho$ of $K$ : Let $\left[\gamma_{1}, \gamma_{2}, \cdots \cdots, \gamma_{n}\right]$ be a basis of $\mathfrak{b}$, then $\left[\alpha_{1}, \alpha_{2}, \cdots \cdots, \alpha_{n}\right], \alpha_{i}=\operatorname{\rho r}_{i}(1 \leqq i \leqq n)$ is a basis of $a$.

This lemma is trivial in the rational number field and imaginary quadratic number fields. We accordingly assume that $K$ is the other field.

If $\left(\varepsilon_{1}, \varepsilon_{2}, \cdots \cdots, \varepsilon_{r}\right)\left(r=n_{1}+n_{2}-1\right)$ is a system of fundamental units, there exists a system of real numbers $\left(x_{1}, x_{2}, \cdots \cdots, x_{r}\right)$ such that

$$
\frac{\left|\rho^{(j)}\right|}{\sqrt[n]{|N \rho|}}=\exp \left(\sum_{\nu=1}^{r} x_{\nu} \log \left|\varepsilon^{\nu}{ }_{(j)}\right|\right) \quad(1 \leqq j \leqq n) .
$$

Multiplying $\rho$ by a suitable unit, we may assume the inequality $0 \leqq x_{\nu}<1(1 \leqq \nu \leqq n)$. Then we obtain

$$
\left|\rho^{(j)}\right| \leqq \sqrt[n]{|N \rho|} \exp \left(\sum_{\nu=1}^{r}|\log | \varepsilon_{(j)}^{\nu}||\right) \leqq c_{3}^{n} \mid \overline{|N \rho|}
$$

and

$$
\left|\rho^{(j)}\right| \geqq \sqrt[n]{|N \rho|} \exp \left(-\sum_{\nu=1}^{r}|\log | \varepsilon^{\nu}{ }_{(j)}||\right) \geq c_{4} \sqrt[n]{|N \rho|}
$$

with positive constants $c_{3}$ and $c_{4}$ depending on $K$ alone. Therefore we have

Further, let

$$
c_{3} \sqrt[n]{|N \rho|} \leqq\left|\rho^{(j)}\right| \leqq c_{4} \sqrt[n]{|N \rho|} \quad(1 \leqq j \leqq n)
$$

$$
\left\{\begin{array}{l}
\operatorname{Max}_{1 \leqq i, j \leqq n}\left|\gamma_{(j)}{ }^{i}\right|=M \sqrt[n]{ } / \overline{N \mathrm{~b}} \\
\operatorname{Min}_{1 \leqq i, j \leqq n}\left|\gamma_{(j)}{ }^{i}\right|=m \sqrt{\boldsymbol{n}} \overline{N \mathrm{~b}} .
\end{array}\right.
$$

Then from (4) and (5) we obtain

and

$$
\left|\alpha^{i}{ }_{(j)}\right|=\left|\rho^{(j)} \gamma^{i}{ }_{(j)}\right| \leqq c_{3} \sqrt[n]{|N \rho|} \cdot M^{n} \sqrt{N \mathfrak{b}}=\left(c_{3} M\right) \sqrt[n]{N b} \quad(1 \leqq i, j \leqq n)
$$

$$
\left|\alpha^{i}\left({ }^{j}\right)\right| \geqq\left(c_{4} m\right) \sqrt[n]{N a}
$$

$(1 \leqq i, j \leqq n)$

Because of the finiteness of the class number, we have the proof.

Lemma 4 . Let $\mathfrak{p}$ be a prime ideal of $K$. Then there exists an integer $\pi \in \mathfrak{D}$ with the conditions that

$$
\begin{gathered}
\mathfrak{p} \| \pi \\
c_{1} \sqrt[n]{N_{p}} \leqq\left|\pi^{(j)}\right| \leqq c_{2} \sqrt[n]{N p} \quad(1 \leqq j \leqq n)
\end{gathered}
$$

Proof. Let $\left[\pi_{1}, \pi_{2}, \cdots \cdots, \pi_{n}\right]$ be a basis of $\mathfrak{p}$. By lemma 3 we may assume that

$$
c_{1} \sqrt[n]{N \mathfrak{p}} \leqq\left|\pi^{i}(j)\right| \leqq c_{2} \sqrt[n]{N \mathfrak{p}}
$$

with positive constants $c_{1}$ and $c_{2}$ depending only on $K$. Here we have at least one $\pi_{i}$ $(1 \leqq i \leqq n)$ such that $\pi_{i} \notin \mathfrak{p}^{2}$. If otherwise, let $\pi_{1}, \pi_{2}, \cdots \cdots, \pi_{n} \in \mathfrak{p}^{2}$, then we have $\mathfrak{p}^{2} \mid \mathfrak{p}=\left[\pi_{1}\right.$, $\left.\cdots \cdots, \pi_{n}\right]$. Hence we have $\pi_{i}$ such that

Hence the lemma follows by taking $\pi=\pi_{i}$.

$$
\pi_{i} \in \mathfrak{p}, \pi_{i} \notin \mathfrak{p}^{2} \text {. }
$$

Lemma 5 . Let a be an integral ideal of $K$ and 5 be an arbitrary residue class mod $a$. Then we can take an integral number $\lambda \in 0$ such that 


$$
\lambda \in\left(5,\left|\lambda^{(j)}\right|<c_{1} \sqrt[n]{N a .}\right.
$$

$(1 \leqq j \leqq n)$

with a positive constant $c_{1}$ depending on $K$ alone.

Proof. Let $\left[\alpha_{1}, \alpha_{2}, \cdots \cdots, \alpha_{n}\right]$ be a basis of a with the conditions of lemma 3 , i.e.,

$$
c_{2} \sqrt[n]{N a} \leqq\left|\alpha^{i}{ }_{(j)}\right| \leqq c_{3} \sqrt[n]{N a}
$$

$(1 \leqq i, j \leqq n)$.

Let $\lambda_{0}$ be an element of $\left(5\right.$. Further, let $\left(x_{1}, x_{2}, \cdots \cdots, x_{n}\right)$ be a real solution satisfying the system of equations

$$
\begin{array}{lr}
\alpha^{1}{ }_{(p)} x_{1}+\cdots \cdots+\alpha^{n}{ }_{(p)} x_{n}=\lambda^{0}{ }_{(p)} & \left(1 \leqq p \leqq n_{1}\right) \\
R\left(\alpha^{1}{ }_{\left(n_{1}+q\right)}\right) x_{1}+\cdots \cdots+R\left(\alpha^{n}{ }_{\left(n_{1}+q\right)}\right) x_{n}=R\left(\lambda^{0}{ }_{\left(n_{1}+q\right)}\right) & \\
I\left(\alpha^{1}{ }_{\left(n_{1}+q\right)}\right) x_{1}+\cdots \cdots-I\left(\alpha^{n}{ }_{\left(n_{1}+q\right)}\right) x_{n}=I\left(\lambda^{0}{ }_{\left(n_{1}+q\right)}\right) & \left(n_{1}+1 \leqq q \leqq n_{1}+n_{2}\right) .
\end{array}
$$

Then, for this real solution, we have

If we let

$$
\alpha^{1}{ }_{(i)} x_{1}+\cdots \cdots+\alpha^{n}{ }_{(i)} x_{n}=\lambda^{0}{ }_{(i)}
$$

and

$$
\alpha=\alpha_{1}\left[x_{1}\right]+\alpha_{2}\left[x_{2}\right]+\cdots \cdots+\alpha_{n}\left[x_{n}\right],
$$

$\alpha$ is an element of $a$, and

$$
\lambda_{0}-\alpha=\lambda,
$$$$
\lambda_{0} \equiv \lambda \bmod a
$$

satisfying the inequalities of our lemma. Therefore we obtain the proof.

Let $R$ be a sufficiently large positive number satisfying the conditions such that

i) $R>\operatorname{Max}\left(\mathrm{k},\left(2^{n} \theta^{-1}\right)^{1 / k}\right),(0<\theta \leqq 1)$,

ii ) We obtain the prime ideal theorem (lemma 1 ).

Obviously we have

$$
\frac{1}{1+\theta} P_{0}^{1 / k}>\frac{1}{1+\theta}(2 R)^{n\left(1+\frac{1}{k-1}\right)^{\tau-1}}>\frac{2}{1+\theta} R^{n}>R .
$$

Hence, by lemma 2 and (1), we obtain a prime ideal $p_{1}$ such that

$$
\frac{1}{1+\theta} P_{0}^{1 / k} \leqq N p_{k 1}<P_{0}^{1 / k} \text {. }
$$

Furthermore, by lemma 4, we obtain an integral number $\pi_{1} \in \mathfrak{v}$ such that

$$
\begin{gathered}
\mathfrak{p}_{1}|| \pi_{1} \\
c_{1,1} N \mathfrak{p}_{1}^{1 / n}<\left|\pi^{(j)}\right|<c_{1,2} N \mathfrak{p}_{1}^{1 / n}
\end{gathered}
$$

with positive constants $c_{1,1}$ and $c_{1,2}$ depending only on $K$. Here we may assume that

Let

and $Q^{1}{ }_{n}=P_{1}$.

$$
0<c_{1,1} \leqq 1, \quad 1 \leqq c_{1,2} \text {. }
$$

$$
Q_{1}=\left[\frac{2}{c_{1,1}}\left(\frac{P_{0}}{N p_{1}}\right)^{1 / n}+1\right]
$$

Then obviously we have

$$
\left(\frac{2}{c_{1,1}}\right)^{n} \frac{P_{0}}{N p_{1}}<P_{1} \leqq\left(\left(\frac{2}{c_{1,1}}\right)\left(\frac{P_{0}}{N p_{1}}\right)^{1 / n}+1\right)^{n} .
$$

Using $P_{1}, Q_{1}$ and $\mathfrak{p}_{1}$, let us define inductively $P_{\nu}, Q_{\nu}$ and $\mathfrak{p}_{\nu}(2 \leqq \nu \leqq k)$.

Let $p_{\nu}$ be a prime ideal, satisfying the condition such that

$$
\frac{1}{1+\theta} P_{\nu-1}^{1 / k} \leq N p_{\nu}<P_{\nu-1}^{1 / k} \text {. }
$$

Let $\pi_{\nu}$ be an integral number of lemma 4 , satisfying the conditions such that

$$
\begin{gathered}
\mathfrak{p}_{\nu}|| \pi_{\nu}, \\
c_{\nu, 1} N \mathfrak{p}_{\nu}^{1 / n}<\left|\pi_{\nu}^{(j)}\right|<c_{\nu, 2} N \mathfrak{p}_{\nu}^{1 / n}
\end{gathered}
$$


with positive constants $c_{\nu, 1}$ and $c_{\nu, 2}$ depending only on $K$. We may assume that $0<c_{\nu, 1} \leqq 1, \quad 1 \leqq c_{\nu, 2}$.

Let $P_{\nu}, Q_{\nu}$ be positive integers, where

$$
\left[\frac{2}{c_{\nu, 1}}\left(\frac{P_{\nu-1}}{N p_{\nu}}\right)^{1 / n}+1\right]=Q_{\nu}, Q_{\nu}^{n}=P_{\nu}
$$

Finally let

$$
\left\{\begin{array}{l}
\widetilde{c}_{1}=\operatorname{Min}\left(c_{1,1}, c_{2,1}, \cdots \cdots, c_{\tau, 1}\right), \\
\widetilde{c}_{2}=\operatorname{Max}\left(c_{1,2}, c_{2,2}, \cdots \cdots, c_{\tau, 2}\right),
\end{array}\right.
$$

then we obtain

$$
0<\widetilde{c}_{1} \leqq 1, \quad 1 \leqq \widetilde{c}_{2}
$$

Lemma 6 . For all $\nu(1 \leqq \nu \leqq \tau)$ we obtain as follows:

$$
\begin{gathered}
N \mathfrak{p}_{\nu}>k^{n}, \\
\frac{1}{1+\theta} P^{\nu}{ }_{1 / k}>R, \\
N \mathfrak{p}_{\nu} \cdot P_{\nu}>P_{\nu-1}, \\
P_{\nu} \leqq\left(\frac{2}{c_{1}}\right)^{\nu n}(1+2 \theta)^{\nu} P_{0}\left(1-\frac{1}{k}\right)^{\nu} \\
\left.P_{\nu} \geqq P_{0}^{\left(1-\frac{1}{k}\right.}\right)^{\nu}
\end{gathered}
$$

Remark. By $(20) \sim(23)$ and lemma 2 , we know the existence of the ideals $\mathfrak{p}_{\nu}(2 \leqq \nu \leqq \tau)$.

ProOF OF LEMMA 6.

i) Proof of (21). By (10) we know

$$
\left(\frac{2}{c_{\nu, 1}}\right)^{n}\left(\frac{P_{\nu-1}}{N \mathfrak{p}_{\nu}}\right)<P_{\nu} \leqq\left(\frac{2}{c_{\nu, 1}}\left(\frac{P_{\nu-1}}{N \mathfrak{p}_{\nu}}\right)^{1 / n}+1\right)^{n}
$$

and from the hypothesis $2 / c_{\nu, 1}>1$ we obtain

$$
\frac{P_{\nu-1}}{N p_{\nu}}<P_{\nu} \text { for all } \nu(1 \leqq \nu \leqq \tau) \text { q.e.d. }
$$

ii) Proof of (23). When $\nu=1$, this result is obviously true:

$$
P_{1} \geq\left(\frac{2}{c_{1,1}}\right)^{n} \frac{P}{N p_{1}} \geq P_{0}^{1-\frac{1}{k}} \text {. }
$$

Suppose that (23) is true for $\nu \leqq i-1(i \geqq 2)$. By (12) and (16) and the hypothesis of the above, we obtain

$$
\begin{aligned}
\left.P_{i \geq(} c_{c_{i, 1}}\right)^{n}\left(\frac{P_{i-1}}{N \mathfrak{p}_{i}}\right) & \geqq P_{i-1}^{1-\frac{1}{k}} \\
& \geq P_{0}^{\left(1-\frac{1}{k}\right)^{i}},
\end{aligned}
$$

and therefore (23) is proved.

iii) Proof of (19) and (20). By (12) and (16), we obtain

$$
\begin{aligned}
N \mathfrak{p}_{\nu} & \geqq \frac{1}{1+\theta} P_{\nu-1}^{1 / k} \geqq \frac{1}{1+\theta}\left(\frac{2}{c_{\nu-1,1}}\right)^{n / k}\left(\frac{P_{\nu-2}}{N \mathfrak{p}_{\nu-1}}\right)^{1 / k} \\
& \geqq \frac{1}{1+\theta} P_{\nu-2}^{-\frac{1}{k}\left(1-\frac{1}{k}\right)} .
\end{aligned}
$$


Furthermore by (23):

$$
\geqq \frac{1}{1+\theta} P_{0}^{-\frac{1}{k}}\left(1-\frac{1}{k}\right)^{\nu-1}
$$

By (1) and (2)

$$
\begin{aligned}
& \geqq \frac{1}{1+\theta}\left((2 R)^{\left.n k\left(1+\frac{1}{k-1}\right)^{\nu-1}\right) \frac{1}{k}\left(1-\frac{1}{k}\right)^{\nu-1}}\right. \\
& =\frac{1}{1+\theta}(2 R)^{n} \geqq R^{n} \geqq\left\{\begin{array}{l}
R \\
k^{n} .
\end{array}\right.
\end{aligned}
$$

q.e.d.

iv) Proof of (22). By (23) and (2) we know that

$$
\begin{aligned}
P_{\nu-1}^{1 / n} \geqq P_{0}^{\frac{1}{n}\left(1-\frac{1}{k}\right)^{\nu}} & \geqq(2 R)^{k\left(1+\frac{1}{k-1}\right)^{\nu}\left(1-\frac{1}{k}\right)^{\nu}} \\
& =(2 R)^{k} .
\end{aligned}
$$

By the hypothesis (1) of $R$, we obtain

$$
P_{\nu-1}^{\frac{1}{n}\left(1-\frac{1}{k}\right)} \geqq\left(2\left(2^{n} \theta^{-1}\right)^{1 / k}\right)^{k} \geqq\left(2^{n+1} \theta^{-1}\right) .
$$

Therefore from the definition of $P_{\nu}$ we obtain

$$
\begin{aligned}
P_{\nu} & \leqq\left(\frac{2}{c_{\nu, 1}}\left(\frac{P_{\nu-1}}{N \mathfrak{p}_{\nu}}\right)^{1 / n}+1\right)^{n} \\
& \leqq\left(\frac{2}{c_{\nu, 1}}\right)^{n} \frac{P_{\nu-1}}{N \mathfrak{p}_{\nu}}+2^{n}\left(\left(\frac{2}{c_{\nu, 1}}\right)\left(\frac{P_{\nu-1}}{N \mathfrak{p}_{\nu}}\right)^{1 / n}\right)^{n-1} \\
& \leqq\left(\frac{2}{\widetilde{c}_{1}}\right)^{n} P_{\nu-1}^{1-\frac{1}{k}}\left\{(1+\theta)+2^{n}(1+\theta)^{1-\frac{1}{n}} P_{\nu-1}^{-\frac{1}{n}}, 1-\frac{1}{k}\right\} \\
& \leqq\left(\frac{2}{\widetilde{c}_{1}}\right)^{n} P_{\nu-1}^{1-\frac{1}{k}}\left\{(1+\theta)+2^{n+1-1 / n} P_{\nu-1}^{-\frac{1}{n}}\left(1-\frac{1}{k}\right)\right\} \\
& \leqq\left(\frac{2}{\widetilde{c}_{1}}\right)^{n}(1+2 \theta) P_{\nu-1}^{1-\frac{1}{k}} \leqq \ldots \ldots \\
& \left.\leqq\left(\frac{2}{\widetilde{c}_{1}}\right)^{n \nu}(1+2 \theta)^{\nu} P_{0}^{\left(1-\frac{1}{k}\right.}\right)^{\nu}
\end{aligned}
$$

Lemma 7. Let $k \geq 2$ and let $\nu_{1}, \nu_{2} \cdots \cdots, \nu_{k}$ be integers in $\mathfrak{o}$. Let $\mathfrak{p}$ be a prime ideal with $N \mathfrak{p}>k^{n}$. By lemma 4 we may take an integer $\pi \in \mathfrak{D}$ satisfying the conditions such that

$$
\begin{gathered}
\mathfrak{p} \| \pi, \\
c_{1} N \mathfrak{p}^{1 / n} \leq\left|\pi^{(j)}\right| \leq c_{2} N \mathfrak{p}^{1 / n} \\
0<c_{1} \leqq 1, \quad 1 \leqq c_{2},
\end{gathered}
$$$$
(1 \leqq j \leqq n)
$$

where $c_{1}$ and $c_{2}$ depend only on $K$.

Let $\lambda_{1}, \lambda_{2}, \cdots \cdots, \lambda_{k}$ be integers in $\mathfrak{D}$ satisfying the conditions such that

$$
\begin{aligned}
& \lambda_{i} \neq \lambda_{j} \quad(i \neq j) \quad(\bmod \mathfrak{p}) \\
& 0<\lambda_{\nu}<m N \mathfrak{p}^{k / n}
\end{aligned} \quad(1 \leqq \nu \leqq k)
$$

with a positive number $m \geqq 1$. Let $T$ denote the number of solutions of the system of congruences 


$$
\left\{\begin{aligned}
\lambda_{1}+\lambda_{2}+\cdots \cdots+\lambda_{k} & \equiv \nu_{1}(\bmod \mathfrak{p}) \\
\lambda_{2}{ }^{2}+\lambda_{2}{ }^{2}+\cdots \cdots+\lambda_{2}{ }^{2} & \equiv \nu_{2}\left(\bmod \mathfrak{p}^{2}\right) \\
\cdots \cdots \cdots \cdots \cdots \cdots \cdots \cdots & \\
\lambda_{k}{ }^{k}+\lambda_{k}{ }^{k}+\cdots \cdots+\lambda_{k}{ }^{k} & \equiv \nu_{k}\left(\bmod \mathrm{p}^{k}\right) .
\end{aligned}\right.
$$

Then the following estimate holds:

$$
T \ll k !\left(c_{3}^{k} m\right)^{n k} N p^{\frac{1}{2} k(k-1)}
$$

with positive constant $c_{3}$ depending only on $K$.

Proof. If $\lambda_{j i}(1 \leqq i, j \leqq k)$ take from a fixed complete system of incongruent residues $(\bmod \mathfrak{p})$, then by lemma 5 , we may assume that

$$
0<\lambda_{j i}<c_{4} \sqrt[n]{N p}
$$

Furthermore, by (23) we may assume the following inequalities

$$
0<\lambda_{j i}<c_{5} \pi, c_{5}>1 \text {, }
$$

Let us write the number $\lambda_{j}(1 \leqq j \leqq k)$ in the form

$$
\lambda_{j}=\lambda_{j 0}+\lambda_{j 1} \pi+\cdots \cdots+\lambda_{j, k-1} \pi^{k-1}+\lambda_{j, k} \pi^{k}
$$

with the conditions (28).

By (23) and (28), we obtain

$$
\begin{aligned}
\left|\lambda_{j}-\lambda_{j, k} \pi^{k}\right| & \leqq c_{5}|\pi|+\cdots \cdots+c_{5}|\pi|^{k} \\
& =c_{5}|\pi| \frac{|\pi|^{k}-1}{|\pi|-1}<2 c_{5}|\pi|^{k} \\
& <2 c_{5} c_{2}{ }^{k} N \mathfrak{p}^{k / n} .
\end{aligned}
$$

Therefore

$$
\begin{aligned}
\left|\lambda_{j, k}\right|\left|\pi^{k}\right| \leqq\left(\left|\lambda_{j}\right|+2 c_{5} c_{2}{ }^{k} N \mathfrak{p}^{k / n}\right) \\
\leqq m N \mathfrak{p}^{k / n}+2 c_{5} c_{2}{ }^{k} N \mathfrak{p}^{k / n} .
\end{aligned}
$$

Hence by (23)

$$
\begin{aligned}
\left|\lambda_{j, k}\right| & \leqq \frac{m+2 c_{5} c_{2}{ }^{k}}{c_{1}{ }^{k}} \leqq \frac{3 c_{5} c_{6}{ }^{k}}{c_{1}{ }^{k}} m \\
& \leqq c_{3}{ }^{k} m
\end{aligned}
$$

where $c_{3}>2$.

Note that in agreement with the supplementary condition (24) stated in the lemma, we have

$$
\lambda_{i 0} \neq \lambda_{j 0} \quad(\bmod p)
$$

for $i \neq j$.

It is easy to see that the numbers $\lambda_{11}, \lambda_{21}, \cdots \cdots, \lambda_{n 1}$ satisfy the system of congruences

$$
\begin{aligned}
& \lambda_{10}+\lambda_{20}+\cdots \cdots+\lambda_{k 0} \equiv \nu_{1} \\
& \lambda_{10}^{2}+\lambda_{20}^{2}+\cdots \cdots+\lambda_{k 0}^{2} \equiv \nu_{2} \\
& \lambda_{10}^{k}+\lambda_{20}^{k}+\cdots \cdots+\lambda_{k 0}^{k} \equiv \nu_{k}
\end{aligned}
$$

Denote dy $T_{1}$ the number of this system. Then we have

$$
T_{1} \leqq k ! \text {. }
$$

This result is obviously true when $k=1$. We now proceed by induction on $k$. Suppose that the conclusion is true for $k-1$. Let $\lambda_{k, 1}$ be known and $T_{1}{ }^{(k-1)}$ the number of solutions of the system of congruences 


$$
\left.\begin{array}{c}
\lambda_{10}+\cdots \cdots+\lambda_{k-1,0} \equiv \nu_{1}-\lambda_{k, 0} \\
\cdots \cdots \cdots \cdots \cdots \cdots \cdots \cdots \cdots \cdots \cdots \cdots \cdots \\
\lambda_{10}^{k-1}+\cdots \cdots+\lambda_{k-1,0}^{k-1} \equiv \nu_{k-1}-\lambda_{k, 0}^{k-1}
\end{array}\right\} \quad(\bmod \mathfrak{p})
$$

where $(31): \lambda_{i_{0}} \neq \lambda_{j_{0}}(\bmod \mathfrak{p})(i \neq j)$. By the hypothesis of induction we know that $T^{1}{ }_{(k-1)} \leqq(k-1) !$.

Denote $\widetilde{T}_{1}^{(k-1)}$ the number of solutions of the congruence

$$
\lambda_{10}^{k}+\cdots \cdots+\lambda_{k-1,0}^{k}+\lambda_{k, 0}^{k} \equiv \nu_{k} \quad(\bmod \mathfrak{p}) .
$$

Obviously we have $\widetilde{T}_{i}^{(k-1)} \leqq k$, hence

$$
T_{1} \leqq T_{1}^{(k-1)} \quad \widetilde{T}_{1}^{(k-1)} \leqq(k-1) ! k=k !
$$

Therefore we obtain (33).

Holding one such solution $\left[\lambda_{10}, \lambda_{20}, \cdots \cdots, \lambda_{k, 0}\right] \bmod \mathfrak{p}$ fixed, let us go over from the basic system of congruences to congruences modulo $\mathfrak{p}^{2}$. We have:

$$
\begin{aligned}
\lambda_{1}^{2}+\cdots \cdots+\lambda_{k}^{2} & \equiv\left(\lambda_{10}+\lambda_{1,1} \pi\right)^{2}+\cdots \cdots+\left(\lambda_{k 0}+\lambda_{k, 1} \pi\right)^{2} \\
& \equiv\left(\lambda_{10}^{2}+\cdots \cdots+\lambda_{k 0}^{2}\right)+2 \pi\left(\lambda_{10} \lambda_{11}+\cdots \cdots+\lambda_{k 0} \lambda_{k 1}\right) \quad\left(\bmod p^{2}\right) .
\end{aligned}
$$

By (32), we know that

$$
\lambda_{10} \lambda_{11}+\cdots \cdots+\lambda_{k_{0}} \lambda_{k_{1}} \equiv \nu_{2,1} \quad(\bmod \mathfrak{p}),
$$

and similarly, we obtain the following system of $k-1$ linear congruences in $\lambda_{11}, \cdots$ $\cdots, \lambda_{k, 1}$ :

$$
\left.\begin{array}{c}
\lambda_{10} \lambda_{11}+\cdots \cdots+\lambda_{k 0} \lambda_{k 1} \equiv \nu_{2,1} \\
\cdots \cdots \cdots \cdots \cdots \cdots \cdots \cdots \cdots \cdots \cdots \cdots \cdots \cdots \cdots \cdots \\
\lambda_{10}^{k-1} \lambda_{11}+\cdots \cdots+\lambda_{k 0}^{k-1} \lambda_{k 1} \equiv \nu_{2, k-1}
\end{array}\right\} \quad \begin{gathered}
(\bmod \mathfrak{p}) \\
\nu_{2,1} \cdots \cdots \nu_{2, k-1} \in \mathfrak{D} .
\end{gathered}
$$

Since the numbers $\lambda_{10}, \cdots \cdots, \lambda_{k_{0}}$ are distinct, at least $k-1$ of them are different from zero. To fix our ideas, we shall assume that $\lambda_{10}, \cdots \cdots, \lambda_{k-1,0}$ differ from zero. Then obviously we have

$$
\begin{aligned}
& \Delta_{2}=\left|\begin{array}{c}
\lambda_{10}, \lambda_{20}, \cdots \cdots, \lambda_{k-1,0} \\
\cdots \cdots \cdots \cdots \\
\lambda_{10}^{k-1}, \quad \lambda_{20}^{k-1}, \cdots \cdots, \lambda_{k-1,0}^{k-1}
\end{array}\right| \\
& =\lambda_{10} \lambda_{21} \cdots \cdots \lambda_{k-1,1} \quad \prod_{1 \leqq i<j \leqq k-1}\left(\lambda_{i 1}-\lambda_{j 1}\right) \neq 0 \quad(\bmod \mathfrak{p})
\end{aligned}
$$

and consequently, for any fixed value of $\lambda_{k, 1}$, the number $\lambda_{1,1} \cdots \cdots \lambda_{k-1,1}$ are uniquely determined from system (34). Thus denoting by $T_{2}$ the number of solutions of this system (34), we know that

$$
T_{2}=N p \text {. }
$$

Generally, considering the congruences

$$
\begin{aligned}
\lambda_{1}^{j}+\cdots \cdots+\lambda_{k}^{j} & \equiv \nu_{j} \equiv\left(\lambda_{10}+\lambda_{11} \pi+\cdots \cdots+\lambda_{1, l-1} \pi^{l-1}\right)^{j} \\
& +\cdots \cdots \\
& +\left(\lambda_{k 0}+\lambda_{k 1} \pi+\cdots \cdots+\lambda_{k, l-1} \pi^{l-1}\right)^{j}\left(\bmod \mathfrak{p}^{l}\right) \quad(l \leqq j \leqq k),
\end{aligned}
$$


we obtain a system of $k-l+1$ congruences:

$$
\nu_{j} \equiv \sum_{q=0}^{l-1} f_{j, q} \pi^{q}+j \sum \lambda_{p 0}^{j-1} \lambda_{p, l-1} \pi^{l-1}\left(\bmod \mathfrak{p}^{l}\right)(l-1 \leqq j \leqq k-1),
$$

where $f_{j, q}(0 \leqq q \leqq l-1)$ is a polynomial of $\lambda_{p, q}(1 \leqq p \leqq k, 0 \leqq q \leqq l-2)$ with rational integral coefficients From these congruences, we obviously obtain

$$
\begin{aligned}
& \sum_{p=1}^{k} \lambda_{p 0}^{j-1} \lambda_{p, l-1} \equiv \nu_{l, j-1} \quad(\bmod \mathfrak{p}) \quad(l-1 \leqq j \leqq k-1) . \\
& \nu_{0, j-1} \in \mathrm{D}
\end{aligned}
$$

Here we have used $N p>k^{n}$. Then, denoting by $T_{l}$ the number of solutions of this system, we have similarly

$$
T_{l}=N \mathfrak{p}^{l-1} \quad(1 \leqq l \leqq k) .
$$

Finally, let

$$
\left[\begin{array}{c}
\lambda_{10}, \cdots \cdots \cdots, \lambda_{1, k-1} \\
\cdots \cdots \cdots \cdots \cdots \cdots \cdots \\
\lambda_{k, 0}, \cdots \cdots, \lambda_{k, k-1}
\end{array}\right]
$$

be known and let $T_{k-1}$ be the number of solutions

$$
\left[\begin{array}{c}
\lambda_{1, k} \\
\dot{\cdot} \\
\dot{\cdot} \\
\lambda_{k, k}
\end{array}\right]
$$

of the system of equations (29). Obviously, by (30) we obtain

$$
T_{k-1} \leqq\left(c_{3}^{k} m\right)^{n k} \text {. }
$$

By (33), (35) and (36), we have

$$
\begin{aligned}
T & \leqq T_{1} T_{2} \cdots \cdots T_{k} T_{k+1} \\
& \leqq k ! N \mathfrak{p}^{1+2+\cdots+k}\left(c_{3}^{k} m\right)^{n k} \\
& \leqq k !\left(c_{3}^{k} m\right)^{n k} N \mathfrak{p}^{\frac{1}{2} k(k+1)}
\end{aligned}
$$

The lemma is proved.

[3] Proof of the main theorem. By lemma 4 and lemma 5, we may assume that

and

$$
\lambda_{i}=\varphi_{i}+\xi_{i} \pi_{1}, \mu_{i}=\phi_{i}+\eta_{i} \pi_{1}
$$

$(1 \leqq i \leqq s)$

$$
\begin{gathered}
0<\varphi_{i}, \phi_{i}<c_{1} \pi_{1} \\
c_{2} \sqrt[n]{N \mathfrak{p}_{1}} \leqq\left|\pi^{(j)}\right| \leqq c_{3} \sqrt[n]{N p_{1}}, p_{1}|| \pi_{1},
\end{gathered}
$$

where $\varphi_{i}$ and $\phi_{i}$ run separately through a complete set of residues mod $\mathfrak{p}_{1}$ and are decided uniquely by $\lambda_{i}$ and $\mu_{i}$ respectively.

By (1) and (2), we obtain

(39) $\left(\varphi_{1}+\xi_{1} \pi_{1}\right)^{\nu}+\cdots \cdots+\left(\varphi_{s}+\xi_{s} \pi_{1}\right)^{\nu}=\left(\phi_{1}+\eta_{1} \pi_{1}\right)^{\nu}+\cdots \cdots+\left(\phi_{s}+\eta_{s} \pi_{1}\right)^{\nu}, \quad(1 \leqq \nu \leqq k)$, where

We shall put the system

$$
0<\varphi_{i}+\xi_{i} \pi_{1}, \phi_{i}+\eta_{i} \pi_{1}<Q,
$$

$$
\left\{\varphi_{1}, \cdots \cdots, \varphi_{s}\right\}, \quad\left\{\phi_{1}, \cdots \cdots, \phi_{s}\right\}
$$

in the first class if we can find $k$ distinct $\varphi_{i}, \phi_{i}$. All the remaining systems $\left\{\varphi_{1}, \cdots\right.$ $\left.\cdots, \varphi_{s}\right\},\left\{\phi_{1}, \cdots \cdots, \phi_{s}\right\}$ will be placed in the second class. 
Let $N_{s}{ }^{(1)}(P)$ be the number of solutions of the system of equations (39) with the conditions $(40)$ for $\left\{\varphi_{1}, \cdots \cdots, \varphi_{s}\right\}$ and $\left\{\psi_{1}, \cdots \cdots, \psi_{s}\right\}$ belonging to the first class, and let $N_{s}{ }^{(2)}(P)$ be the number of solutions of (39) with (40) for either $\left\{\varphi_{1}, \cdots \cdots, \varphi_{s}\right\}$ or $\left\{\psi_{1}, \cdots \cdots, \psi_{s}\right\}$ belonging to the second class. By lemma 6, (21), we have obviously

$$
N_{s}(P) \leqq N_{s}\left(N \mathfrak{p}_{1} P_{1}\right)=N_{s}^{(1)}\left(N \mathfrak{p}_{1} P\right)+N_{s}^{(2)}\left(N p_{1} P_{1}\right) .
$$

Let us denote by

$$
\left\{\left(\varphi_{1}, \cdots \cdots, \varphi_{k}\right) \varphi_{k+1}, \cdots \cdots, \varphi_{s}\right\}
$$

the system with distinct $\varphi_{1}, \cdots \cdots, \varphi_{k}$ and arbitrary $\varphi_{k+1}, \cdots \cdots, \varphi_{s}$. We shall call permutations of these systems such systems in which the distinct $\varphi_{j}$ occupy arbitrary places, and not the first $k$ places.

It is obvious to see that every system of the first class is included in the permutation of systems of the form $\left\{\left(\varphi_{1}, \cdots \cdots, \varphi_{k}\right) \varphi_{k+1}, \cdots \cdots, \varphi_{s}\right\}$. Hence the number $N_{s}^{(1)}(P)$ does not exceed the number of solutions of system (39), with variables of the form $\left\{\left(\varphi_{1}, \cdots \cdots, \varphi_{k}\right) \varphi_{k+1}, \cdots \cdots, \varphi_{s}\right\},\left\{\left(\psi_{1}, \cdots \cdots, \psi_{k}\right) \psi_{k+1}, \cdots \cdots, \psi_{s}\right\}$, multiplied by

$$
\left(\begin{array}{c}
s \\
k
\end{array}\right)^{2}={ }_{s} C_{k}^{2} \text {. }
$$

Let $\gamma_{i}(1 \leqq i \leqq n)$ be numbers of $K$ and $x_{i}(1 \leqq i \leqq n)$ be real variables. For $\xi=$ $\sum_{\nu=1}^{n} \gamma_{\nu} x_{\nu}$, we define

$$
\operatorname{trace}(\gamma)=\sum_{i=1}^{n} \gamma^{(i)}, \quad \operatorname{trace}(\xi)=\sum_{i=1}^{n} \xi^{(i)}
$$

and

$$
E(\gamma)=e^{2 \pi i \operatorname{trace}(\gamma)}, \quad E(\xi)=e^{2 \pi i \operatorname{trace}(\xi)} .
$$

Let $\delta$ be the ramification ideal of $K$ and let $\left[\omega_{1}, \cdots \cdots, \omega_{n}\right]$ be an integral basis of $K$. We can choose $\left[\rho_{1}, \cdots \cdots, \rho_{n}\right]$ a basis of $\delta^{-1}$, such that

$$
\operatorname{trace}\left(\rho_{i} \omega_{j}\right)=\left\{\begin{array}{ll}
1 & (i \neq j) \\
0 & (i \neq j)
\end{array} \quad(1 \leqq i, j \leqq n) .\right.
$$

Let $X$ be the whole $n$-dimensional Euclidean space and $U\left(U_{1}, \cdots \cdots, U_{n}\right)$ be the unit cube

We set

$$
\left\{\left(x_{1}, x_{2}, \cdots \cdots, x_{n}\right): 0 \leqq x_{i} \leqq 1(1 \leqq i \leqq n)\right\}
$$

$$
\left\{\begin{aligned}
\alpha_{1}= & \rho_{1} y_{11}+\cdots \cdots+\rho_{n} y_{1 n} \\
& \cdots \cdots \cdots \cdots \cdots \cdots \cdots \\
\alpha_{k}= & \rho_{1} y_{k 1}+\cdots \cdots+\rho_{n} y_{k n}
\end{aligned}\right.
$$

with respect to real variables $y_{\nu 1}, \cdots \cdots, y_{\nu n}(1 \leqq \nu \leq n)$. We use abbreviations for all $\nu(1 \leqq \nu \leqq k)$

and

$$
d y_{\nu}=d y_{\nu 1} \cdots \cdots \cdot d y_{\nu n}
$$

$$
\int_{U_{\nu}} * * d y_{\nu}=\int_{0}^{1} \cdots \cdots \cdots \int_{0}^{1} * * d y_{\nu 1} \cdots \cdots \cdot d y_{\nu n} .
$$

For fixed $\varphi$, we define a trigonometrical sum as follows:

$$
S(\varphi)=\sum_{\substack{\lambda=\varphi+\xi_{\pi 1} \\ 0<\lambda<N \beta_{1} P_{1}}} E\left(\alpha_{1} \lambda+\cdots \cdots+\alpha_{k} \lambda^{k}\right) .
$$


Since, by definition of $D$ :

$$
D^{-1}=\{\rho ; E(\rho \xi)=1 \text { for every } \xi \ni \emptyset\}
$$

we obtain

$$
N_{s}^{(1)}=N_{s}^{(1)}\left(N p_{1} P_{1}\right)=\int_{U_{1}} \cdots \cdots \int_{U_{k}}\left|\sum_{\varphi} S(\varphi)\right|^{2 s} d y_{1} \cdots \cdots \cdot d y_{k},
$$

where $\sum_{\varphi}$ runs through a complete system of residuet mod $\mathfrak{p}_{1}$.

Hence we have

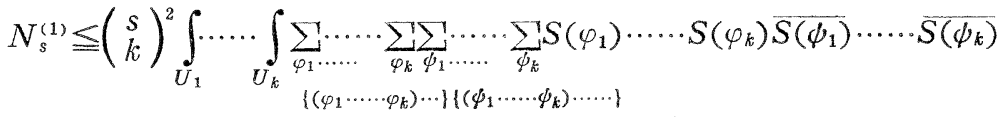

$$
\begin{aligned}
& \times \sum_{\varphi}|S(\varphi)|^{2(s-k)} d y_{1} \cdots \cdots d y_{k} .
\end{aligned}
$$

By Hölder's inequality we have

$$
N_{s}^{(1)} \leqq\left(\begin{array}{c}
s \\
k
\end{array}\right)^{2} N p_{1}{ }^{2(s-k)-1} \int_{U_{1}} \cdots \cdots \int_{U_{k}} A\left(\alpha_{1}, \cdots \cdots, \alpha_{k}\right) d y_{1} \cdots \cdots d y_{k},
$$

where

$$
\begin{aligned}
& A\left(\alpha_{1}, \cdots \cdots, \alpha_{k}\right) \\
& =\left|\sum_{\left(\varphi_{1} \cdots \cdots \varphi_{k}\right) \cdots \cdots} \cdots \sum_{1} S\left(\varphi_{1}\right) \cdots \cdots S\left(\varphi_{k}\right)\right|^{2} \sum_{\varphi}|S(\varphi)|^{2(s-k)}
\end{aligned}
$$

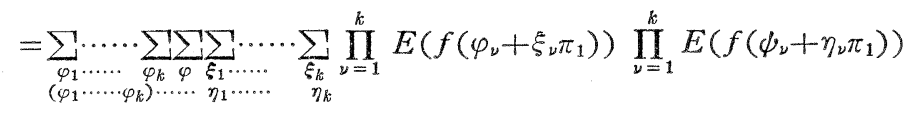

$$
\begin{aligned}
& \times \sum_{\substack{\xi_{k+1} \cdots \cdots . . . \\
\eta_{k+1} \cdots \cdots}} \cdots \sum_{\eta_{s}} \prod_{\nu=k+1}^{s} E\left(f\left(\varphi+\xi_{\nu} \pi_{1}\right)\right) \prod_{\nu=k+1}^{s} E\left(f\left(\varphi+\eta_{\nu} \pi_{1}\right)\right) .
\end{aligned}
$$

Let

$$
A\left(\alpha_{1}, \cdots \cdots, \alpha_{k}\right)=\sum_{\varphi_{\nu}, \psi_{\nu}} \sum_{\nu} \sum_{\varphi} \sum_{\xi_{\nu}} \cdots \sum_{\eta_{\nu}} \sum_{\xi_{\nu}} \cdots \sum_{\eta_{\nu}} E(B)
$$

Then we have

$$
B=\sum_{\nu=1}^{k} f\left(\varphi_{\nu}+\xi_{\nu} \pi_{1}\right)-\sum_{\nu=1}^{k} f\left(\phi_{\nu}+\eta_{\nu} \pi_{1}\right)+\sum_{\nu} \sum_{=k+1}^{s} f\left(\varphi+\xi_{\nu} \pi_{1}\right)-\sum_{\nu \prime}^{s} f\left(\varphi+\eta_{\nu+1} \pi_{1}\right) .
$$

Furthermore,

$$
\int_{U_{1}} \cdots \cdots \int_{U_{k}} A\left(\alpha_{1}, \cdots \cdots, \alpha_{k}\right) d y_{1} \cdots \cdots \cdot d y_{k}
$$

is equal to the number of the system of equations

$$
\sum_{\nu=1}^{k}\left(\varphi_{\nu}+\xi_{\nu} \pi_{1}\right)^{p}-\sum_{\nu=1}^{k}\left(\phi_{\nu}+\eta_{\nu} \pi_{1}\right)^{p}=\sum_{\nu} \sum_{=k+1}^{s}\left(\varphi+\xi_{\nu} \pi_{1}\right)^{p}-\sum_{\nu} \sum_{=k+1}^{s}\left(\varphi+\eta_{\nu} \pi_{1}\right)^{p}
$$

$(1 \leqq p \leqq k)$

with the conditions (40).

For all $p(1 \leqq p \leqq k)$, we have 


$$
\sum_{\nu=1}^{k}\left(\varphi_{\nu}+\xi_{\nu} \pi_{1}\right)^{p}=\sum_{l=0}^{p}\left\{\sum_{\nu=1}^{k}\left(\varphi_{\nu}+\xi_{\nu} \pi_{1}-\varphi_{\nu}\right)^{p-l}\right\}\left(\begin{array}{c}
p \\
l
\end{array}\right) \varphi_{\nu}^{l}
$$

and

$$
\sum_{\nu=k+1}^{s}\left(\varphi+\xi_{\nu} \pi_{1}\right)^{p}=\sum_{l=0}^{k}\left\{\left(\sum_{\nu}^{s} \sum_{=k+1}^{s} \xi_{\nu \prime}^{p-l}\right) \pi_{1}^{p-l}\right\}\left(\begin{array}{l}
p \\
l
\end{array}\right) \varphi^{l} .
$$

By (47) and (48), we find by induction that

$$
\sum_{\nu=1}^{k}\left(\varphi_{\nu}+\xi_{\nu} \pi_{1}-\varphi\right)^{p}-\sum_{\nu=1}^{k}\left(\varphi_{\nu}+\eta_{\nu} \pi_{1}-\varphi\right)^{p}=\pi_{1}^{p} \sum_{\nu \prime}^{s}\left(\xi_{\nu+k}^{p}-\eta_{\nu \prime}^{p}\right), \quad(1 \leqq p \leqq k) .
$$

Let $N^{\prime}(P)$ be the number of solutions of the system of equations (49) with the conditions (40).

Letting

$$
\sum_{\nu \prime=k+1}^{s} \xi_{\nu \prime}^{p}-\sum_{\nu \prime=k+1}^{s} \eta_{\nu^{\prime}}^{p}=\kappa_{p}
$$

we obtain

$$
\sum_{\nu=1}^{k}\left(\varphi_{\nu}+\xi_{\nu} \pi_{1}-\varphi\right)^{p}-\sum_{\nu=1}^{k}\left(\phi_{\nu}+\eta_{\nu} \pi_{1}-\varphi\right)^{p}=\pi_{1}^{p} \kappa_{p}
$$

$(1 \leqq p \leqq k)$

Let us consider the system of equations (51), where $\kappa_{1}, \cdots \cdots, \kappa_{k}$ are arbitrary fixed integers in $\mathrm{D}$.

Let $N^{\prime \prime}\left(\pi_{1} \kappa_{1}, \cdots \cdots, \pi^{k} \kappa_{k}\right)$ be the number of solutions of system (51) and let $T^{\prime}$ be the number of solutions of the system of equations

$$
\sum_{\nu=1}^{k}\left(\varphi_{\nu}+\xi_{\nu} \pi_{1}-\varphi\right)^{p}-\sum_{\nu=1}^{k}\left(\phi_{\nu}+\eta_{\nu} \pi_{1}-\varphi\right)^{p} \equiv 0\left(\bmod \mathfrak{p}_{1}^{p}\right) \quad(1 \leqq p \leqq k) .
$$

By (37) and (38) we may assume that

$$
\varphi_{\nu}, \phi_{\nu}<c_{4} N p_{1}^{1 / n}\left(c_{4}>1\right)
$$

$(1 \leqq \nu \leqq s)$

Hence, by (12) and (53), we obtain

$$
\varphi, \phi<c_{4} N p_{1}^{1 / n}<c_{4} P_{o}^{(1 / k)(1 / n)}=c_{4} Q_{0}^{1 / k}<Q_{0} .
$$

By (40) and (54) we have

By (8) and (55) we have

$$
\left|\xi \pi_{1}\right| \leqq\left|Q_{0}\right|+|\varphi| \leqq 2 Q_{0} .
$$

$$
\eta, \xi<\frac{2 Q_{0}}{c_{1,1} N p_{1}{ }^{1 / n}}<Q_{1}=\left[\frac{2}{c_{1,1}} \frac{Q_{0}}{N p_{1}^{1 / n}}+1\right] .
$$

Therefore by (50), (51) and (52), we obtain

$$
\begin{aligned}
N_{s}^{\prime} & \leqq \sum_{\kappa_{1}} \cdots \cdots \sum_{\kappa_{k}} N_{s}^{\prime \prime}\left(\kappa_{1} \pi_{1}, \cdots \cdots, \kappa_{k} \pi^{k}\right) N_{s-k}\left(\frac{2 Q_{0}}{c_{1,1} N p_{1}^{1 / n}} ; \kappa_{1}, \cdots \cdots \kappa_{k}\right) \\
& \leqq \sum_{\kappa_{1}} \cdots \cdots \sum_{\kappa_{k}} N_{s}^{\prime \prime}\left(\kappa_{1} \pi_{1}, \cdots \cdots, \kappa_{k} \pi^{k}\right) N_{s-k}\left(Q_{1}, \kappa_{1}, \cdots \cdots, \kappa_{k}\right) \\
& \leqq N_{s-k}\left(P_{1}\right) \sum_{\kappa_{1}} \cdots \cdots \sum_{\kappa_{k}} N_{s}^{\prime \prime}\left(\kappa_{1} \pi_{1}, \cdots \cdots, \kappa_{k} \pi^{k}\right) \\
& =N_{s-k}\left(P_{1}\right) T^{\prime} .
\end{aligned}
$$

In the congruences (52) the number of solutions of $\psi_{1}, \cdots \cdots, \psi_{k}, \psi$ is at most $N p_{1}{ }^{k+1}$ and the number of solutions of $\eta_{1}, \cdots \cdots, \eta_{k}$ is at most $P_{1}{ }^{k}$. For any fixed values of 
$\phi_{1}, \cdots \cdots, \phi_{k}, \phi$ and $\eta_{1}, \cdots \cdots, \eta_{k}$ let

$$
\sum_{\nu=1}^{k}\left(\phi_{\nu}+\eta_{\nu} \pi_{1}-\varphi\right)^{p}=\omega_{p}
$$

and

$$
\varphi_{\nu}+\xi_{\nu} \pi_{1}-\varphi=\alpha_{\nu}
$$

$(1 \leqq p \leqq k)$

Then, by (52), (58) and (59), we have

$$
\left\{\begin{aligned}
\alpha_{1}+\alpha_{2}+\cdots \cdots+\alpha_{k} & \equiv \omega_{1}\left(\bmod \mathfrak{p}_{1}\right) \\
\alpha_{1}^{2}+\alpha_{2}^{2}+\cdots \cdots+\alpha_{k}^{2} & \equiv \omega_{2}\left(\bmod \mathfrak{p}_{2}{ }^{1}\right) \\
\cdots \cdots \cdots \cdots \cdots \cdots & \\
\alpha_{1}^{k}+\alpha_{2}^{k}+\cdots \cdots+\alpha_{k}^{k} & \equiv \omega_{k}\left(\bmod \mathfrak{p}_{k}{ }^{1}\right) .
\end{aligned}\right.
$$

Since

$$
\varphi_{\nu}+\xi_{\nu} \pi_{1}=\varphi+\alpha_{\nu}
$$

for any fixed value of $\alpha_{1}, \cdots \cdots, \alpha_{k}, \varphi_{\nu}$ and $\xi_{\nu}(1 \leqq \nu \leqq k)$ are determined uniquely.

By (50) we have

$$
\left|\alpha_{\nu}\right| \leqq\left|\varphi_{\nu}+\xi_{\nu} \pi_{1}\right|+|\varphi| \leqq 2 Q_{0}=\frac{2 Q_{0}}{N p_{1}{ }^{k / n}} N \mathfrak{p}^{1}{ }_{k / n}
$$

Therefore $m$ of lemma 7 is equal to

$$
\begin{aligned}
m & =\frac{2 Q_{0}}{N p_{1}{ }^{k / n}}=2 \frac{Q_{0}}{N p_{1}^{1 / n}} N p_{1}-\frac{k}{n}+\frac{1}{n} \\
& =c_{1,1}\left(\frac{2}{c_{1,1}} \frac{Q_{0}}{N p_{1}^{1 / n}}\right) N p_{1} \frac{1-k}{n} .
\end{aligned}
$$

Hence, by (24) and (61) we have

$$
m<c_{1,1} Q_{1} N p_{1}-\frac{k-1}{n} .
$$

By lemma 7 and (52) we obtain

$$
\begin{aligned}
& T^{\prime} \leqq N p_{1}^{k+1} P_{1}^{k}\left(k !\left(c_{5}^{k} c_{1,1} Q_{1} N p_{1}^{-\frac{k-1}{n}}\right)^{k n} N p_{1}^{\frac{1}{2} k(k-1)}\right) \\
& \leqq k !\left(c_{5}^{k} C_{1,1}\right)^{k n} P_{1}^{k} Q_{1}^{n k} N \mathfrak{p}_{1}^{-k^{2}+k+\frac{1}{2} k(k-1)+k+1} \\
& \leqq k ! c_{6}{ }^{2} n P_{1}{ }^{2 k} N p^{-\frac{1}{2} k(k+1)+2 k+1} .
\end{aligned}
$$

Therefore, by (42), (43), (57) and (63) we finally obtain

$$
\begin{aligned}
& N_{s}{ }^{(1)} \leqq\left(\begin{array}{l}
s \\
k
\end{array}\right)^{2} N p_{1}{ }^{2(s-k)-1} \int_{U_{1}} \cdots \cdots \int_{U_{k}} A\left(\alpha_{1}, \cdots \cdots, \alpha_{k}\right) d y_{1} \cdots \cdots d y_{k} \\
& =\left(\begin{array}{l}
s \\
k
\end{array}\right)^{2} N p_{1}{ }^{2(s-k)-1} N_{s}{ }^{\prime} \\
& \leqq\left(\begin{array}{l}
s \\
k
\end{array}\right)^{2} N p_{1}{ }^{2(s-k)-1} T^{\prime} N_{s-k}\left(P_{1}\right) \\
& \leqq\left(\begin{array}{l}
s \\
k
\end{array}\right)^{2} N \mathfrak{p}_{1}{ }^{2 s-2 k-1} k ! c_{6}^{k 2 n} P_{1}^{2 k} N p_{1}{ }^{-\frac{1}{2} k(k+1)+2 k+1} N_{s-k}\left(P_{1}\right) .
\end{aligned}
$$

Hence 


$$
N_{s}{ }^{(1)} \leqq\left(\begin{array}{c}
s \\
k
\end{array}\right)^{2} k ! c_{6}^{k^{2 n}} P_{1}^{2 k} N p_{1}{ }^{2 s-\frac{1}{2} k(k+1)} N_{s-k}\left(P_{1}\right)
$$

Let us now estimate $N_{s}^{(2)}$. By the definition of $N_{s}^{(2)}$ we obtain

$$
N_{s}^{(2)}=\int_{U_{1}} \cdots \cdots \int_{U_{k}} C\left(\alpha_{1}, \cdots \cdots, \alpha_{k}\right) d y_{1} \cdots \cdots \cdot d y_{k}
$$

with

$$
C\left(\alpha_{1}, \cdots \cdots, \alpha_{k}\right)=\sum_{\varphi_{1}} \cdots \cdots \sum_{\varphi_{s}} \sum_{\psi_{1}} \cdots \cdots \sum_{\psi_{s}} S\left(\varphi_{1}\right) \cdots \cdots S\left(\varphi_{s}\right) \overline{S\left(\phi_{1}\right)} \cdots \cdots \overline{S\left(\phi_{s}\right)},
$$

where the integers $\left\{\varphi_{1}, \cdots \cdots, \varphi_{s}\right\},\left\{\phi_{1}, \cdots \cdots, \phi_{s}\right\}$ vary in such a way that at least one of the systems $\left\{\varphi_{1} \cdots \cdots \varphi_{s}\right\},\left\{\phi_{1} \cdots \cdots \psi_{s}\right\}$ belong to the second class and $\varphi_{\nu}, \psi_{\nu}(1 \leqq \nu \leqq s)$ satisfy the conditions (37), (38) and (40).

Let $M$ be the number of the systems $\left\{\varphi_{1} \cdots \cdots \varphi_{s}\right\}$ such that they belong to the second class. Further $M_{\nu}$ be the number of the systems $\left\{\varphi_{1}, \cdots \cdots, \varphi_{s}\right\}$ in which we can find $\nu$ different $\varphi_{i}(1 \leqq i \leqq s)$. Obviously we have $M_{0}=N \mathfrak{p}_{1}, M_{1}=\left(\begin{array}{c}N p_{1} \\ 2\end{array}\right) 2^{s}, \cdots \cdots$, $M_{s-1}=\left(\begin{array}{l}N p_{1} \\ k-1\end{array}\right)(k-1)^{s}$. From $N p_{1}>k^{n}(k \geq 2)$, we have

$$
\begin{aligned}
M & \leqq M_{0}+M_{1}+\cdots \cdots+M_{k-1} \\
& \leqq N p_{1}+\left(\begin{array}{c}
N p_{1} \\
2
\end{array}\right) 2^{s}+\cdots \cdots+\left(\begin{array}{c}
N p_{1} \\
k-1
\end{array}\right)(k-1)^{s} \\
& \leqq\left(\begin{array}{c}
N p_{1} \\
k-1
\end{array}\right) k(k-1)^{s}
\end{aligned}
$$

$$
\leqq N p_{1}^{k-1} k^{s} \text {. }
$$

Taking $\left\{\psi_{1}, \cdots \cdots, \phi_{s}\right\}$ arbitrarily, the number of the systems $\left\{\phi_{1}, \cdots \cdots, \phi_{s}\right\}$ is $N p_{s}{ }^{1}$. Hence, by (66) and (67) we have

$$
\begin{aligned}
\left|C\left(\alpha_{1}, \cdots \cdots, \alpha_{k}\right)\right| & \leqq N \mathfrak{p}_{1}^{k-1} \cdot k^{s} \cdot N \mathfrak{p}_{1}^{s} \operatorname{Max}_{1 \leqq i \leqq s}\left|S\left(\varphi_{\nu}\right)\right|^{2 s} \\
& \leqq k^{s} N \mathfrak{p}_{1}^{s+k-1} \sum_{\varphi}|S(\varphi)|^{2 s} \\
& \leqq k^{s} N p_{1}^{s+k-1} P_{1}^{2 k} \sum_{\varphi}|S(\varphi)|^{2(s-k)} .
\end{aligned}
$$

Further, by (65) and (68) we have

$$
N_{s}^{(2)} \leqq 2 k^{s} P_{1}^{2 k} N p_{1}^{s+k-1} N_{s-k}\left(P_{1}\right)
$$

Since by (3)

it follows that

$$
s \geqq \frac{1}{2} k(k+1)+\tau(k-1), \quad(\tau=1)
$$

$$
N p_{1}^{2 s-\frac{1}{2} k(k+1)} \geq N p_{1}^{s+k-1}
$$

and

$$
\begin{aligned}
\left(\begin{array}{c}
s \\
k
\end{array}\right)^{2} k ! & \geq(s(s-1) \cdots(s-k+1))^{2} / k ! \\
& \geqq(s-k+1)^{2 k} / k ! \\
& \geq\left(\left(\frac{1}{2}(k+1)\right)^{2 k} / 2 k !\right) 2 k^{2 k}
\end{aligned}
$$


(71)

$$
\geq 2 k^{2 k} \text {. }
$$

Hence by (64), (69), (70) and (71) we have

$$
N_{s}^{(2)}<N_{s}^{(1)} .
$$

Therefore, by (41) and (64), we have

Hence

$$
\begin{gathered}
N_{s}(P) \leqq N_{s}^{(1)}+N_{s}^{(2)} \leqq 2 N_{s}^{(1)} \\
\leqq 2 c_{6}^{k^{2} n}\left(\begin{array}{c}
s \\
k
\end{array}\right)^{2} \cdot k ! P_{1}^{2 k} N p_{1}^{2 s-\frac{1}{2} k(k+1)} N_{s-k}\left(P_{1}\right) .
\end{gathered}
$$

$$
N_{s}(P) \leqq D_{1} P^{2 k} N p_{1}^{2 s-\frac{1}{2} k(k+1)} N_{s-k}\left(P_{1}\right),
$$

where

$$
D_{1}=c_{6}^{k^{2} n}\left(\begin{array}{l}
s \\
k
\end{array}\right)^{2} k !, c_{6}>2
$$

Applying this inequality $\tau$ times, we have

$$
\begin{aligned}
N_{s}(P) \leqq & D_{1} D_{2}\left(P_{1} P_{2}\right)^{2 k} N \mathfrak{p}_{1}{ }^{2 s-\frac{1}{2} k(k+1)} N \mathfrak{p}_{2}{ }^{2(s-k)-\frac{1}{2} k(k+1)} N{ }_{s-2 k}\left(P_{2}\right) \\
\leqq & \cdots \cdots \\
\leqq & D_{1} D_{2} \cdots \cdots D_{\tau}\left(P_{1} P_{2} \cdots \cdots P_{\tau}\right)^{2 k}\left(N p_{1} N p_{2} \cdots N \mathfrak{p}_{\tau}\right)^{2 s-\frac{1}{2} k(k+1)} \\
& \quad \times\left(N p_{2} N p_{3}^{2} \cdots N p_{\tau}^{\tau-1}\right)^{-2 k} P_{\tau}{ }^{2(s-\tau k)} .
\end{aligned}
$$

By lemma 6 , we have

$$
\begin{aligned}
\left(P_{1} P_{2} \cdots \cdots P_{\tau}\right)^{2 k} & \leqq\left(N \mathfrak{p}_{2} P_{2} P_{2} \cdots \cdots P_{\tau}\right)^{2 k} \\
& \leqq \cdots \cdots \\
& \leqq\left(N \mathfrak{p}_{2} N \mathfrak{p}_{2}{ }^{3} \cdots \cdots N \mathfrak{p}_{\tau-1}{ }^{\tau}\right)^{2 k} P_{\tau}^{2 k \tau}
\end{aligned}
$$

By (12) and (22), we have

$$
\begin{aligned}
N \mathfrak{p}_{1} N \mathfrak{p}_{2} \cdots \cdots N \mathfrak{p}_{\tau} \leqq & \left(P_{0} P_{1} \cdots \cdots P_{\tau-1}\right)^{1 / k} \\
& \leqq\left(\left(\frac{2}{\widetilde{c}_{1}}\right)^{n}(1+2 \theta)\right)^{\frac{1}{k}(1+2+\cdots \cdots+\tau-1)} P_{0}^{\frac{1}{k}\left(1+\left(1-\frac{1}{k},+\cdots \cdots+1-\frac{1}{k}{ }^{\tau-1}\right)\right.} \\
& \leqq\left(\left(\frac{2}{\widetilde{c}_{1}}\right)^{n}(1+2 \theta)\right)^{\frac{1}{2 k} \tau(\tau-1)} P_{0}^{\frac{1}{k} \frac{1-(1-1 / k)^{\tau}}{1-(1-1 / k)}} \\
& \leqq\left(\left(\frac{2}{\widetilde{c}_{1}}\right)^{n}(1+2 \theta)\right)^{\frac{1}{2 k} \tau(\tau-1)} P_{0}^{1-(1-1 / k)^{\tau}}
\end{aligned}
$$

Furthermore, by (22) we obtain

$$
\begin{gathered}
P_{\tau}^{2(s-\tau k)}=P_{\tau}^{2 s} P_{\tau}^{-2 \tau k} \\
\leqq\left(\left(\frac{2}{\widetilde{c}_{1}}\right)^{n}(1+2 \theta)\right)^{2 s \tau} P^{2 s(1-1 / k)^{\tau}} P^{-2 \tau k}
\end{gathered}
$$

Summing up the results of $(74),(75),(76)$ and (77), we obtain

$$
N_{\mathrm{s}}(P) \leqq D_{1} D_{2} \cdots \cdots D_{\tau}\left(\left(N p_{2} N p_{2}{ }^{3} \cdots \cdots N p_{\tau}^{\tau-1}\right)^{2 k} P_{\tau}^{2 k \tau}\right)
$$




$$
\begin{aligned}
& \times\left(\left(\left(\frac{2}{\widetilde{c}_{1}}\right)^{n}(1+2 \theta)\right)^{\frac{1}{2 k} \tau(\tau-1)} P^{1-\left(1-\frac{1}{k}\right)^{\tau}}\right)^{2 s-\frac{1}{2} k(k+1)} \\
& \times\left(N \mathfrak{p}_{2} N \mathfrak{p}_{3}^{2} \cdots \cdots N \mathfrak{p}_{\tau}^{\tau-1}\right)^{-2 k}\left(\left(\frac{2}{\widetilde{c}_{1}}\right)^{n}(1+2 \theta)\right)^{2 s \tau} P^{2 s(1-1 / k)^{\tau}} P_{\tau}^{-2 s \tau} \\
& \leqq D_{1} D_{2} \cdots \cdots D_{\tau}\left(\left(\frac{2}{\widetilde{c}_{1}}\right)^{n}(1+2 \theta)\right)^{2 s \tau+\frac{1}{2 k} \tau(\tau-1)\left(2 s-\frac{1}{2} k(k+1)\right)} \\
& \times P^{2 s-\frac{1}{2} k(k-1)+\frac{1}{2} k(k+1)(1-1 / k)^{\tau}}
\end{aligned}
$$

(78)

$$
=D(1+2 \theta)^{\sigma} P^{\eta}
$$

where

$$
\begin{aligned}
& \sigma=2 s \tau+\frac{1}{k} \tau(\tau-1) s-\frac{1}{4} \tau(\tau-1)(k+1), \\
& \eta=2 s-\frac{1}{2} k(k+1)+\frac{1}{2} k(k+1)(1-1 / k)^{\tau}
\end{aligned}
$$

and by (73)

$$
\begin{aligned}
& D\left.=D_{1} D_{2} \cdots \cdots D_{\tau}\left(\frac{2}{\widetilde{c}_{1}}\right)^{n\left(2 s \tau+\frac{1}{2 k} \tau(\tau-)\left(2 s-\frac{1}{2} k(k+1)\right.\right.}\right) \\
&\left.\leqq c_{7}\right) \\
& \times\left(\left(\begin{array}{l}
s \\
k
\end{array}\right)\left(\begin{array}{c}
\left.s-2 k+2 s \tau+\frac{s}{k} \tau(\tau-1)-\frac{1}{4} \tau(\tau-1)(k+1)\right) \\
k
\end{array}\right) \cdots\left(\begin{array}{c}
s-(\tau-1) k \\
k
\end{array}\right)\right)^{2}(k !)^{\tau} \\
& \leqq c_{7} \\
& \leqq \\
&\left.\leqq c_{7}+2 s+\frac{(\tau-1) s}{k}-\frac{1}{4}(\tau-1)(k+1)\right) \tau n \\
&
\end{aligned}
$$

with positive constant $c_{7}$ depending only on $K$.

By (78), (79), (80) and (81) we have

$$
\begin{aligned}
N_{s}(P) \leqq & c_{7} \\
& \times(1+2 \theta)^{2 s \tau+\frac{1}{k} \tau(\tau-1) s-\frac{1}{4} \tau(\tau-1)(k+1)} \cdot s^{2 \tau k} \\
& \times P^{2 s-\frac{1}{2} k(k+1)+\frac{1}{2} k(k+1)(1-1 / k)^{\tau}}
\end{aligned}
$$

Hence the proof of the main theorem is complete.

\section{Bibliography}

[1] И. М. Виноградов: Метод ртигонометрических сумм в теории чисел, Избранные Труды, москва, 1952.

[2] L.K. Hua: An improvement of Vinogradov's mean value theorem and several applications, 
Quart. J. Math. Oxford Ser. 20 (1949), p. 48-61.

[3 ] L.K. Hua: Additive Primzahltheorie, B.G. Teubner Verlags, 1959.

[4] L.K. Hua: Die Abschätzung von Exponentialsummen und ihre Anwendung in der Zahlentheorie, Enzykl. Math. Wiss. Bd. 12, Heft 13, Teil I (1959).

[5] A.A. Karacuba, N.M. Korobov: On the mean value theorem, Sov. Math. 4 (1963), p. 250-254.

[6] O. Körner: Über Mittelwerte trigonometrischen Zahlkörpern, Math. Ann. Bd. 147 (1962), p. 205-239.

[7] E. Landau: Einführung in die elementare und analytische Theorie der algebraischen Zahlen und der Ideale, Zweite Auflage, Leipzig, 1927.

[8] H. Rademacher: Über die Anwendung der Biggo Brunschen Methode auf die Theorie der algebraischen Zahlkörper, S-B. Preuss. Akad., Berlin (1923), p. 211-218.

DEPARTMENT OF MATHEMATICS, KANAZAWA UNIVERSITY 